\title{
SGLT-2 inhibitors as adjunctive to insulin therapy in type 1 diabetes
}

\section{ABSTRACT}

The absolute insulin deficiency that occurs in type 1 diabetes mellitus (T1DM) is associated with the need for intensive functional insulin therapy as the only appropriate treatment model. In the recent years, introduction of new classes of glucose-lowering drugs has led to an increasing interest in adjunct therapies for T1DM. These therapies are designed to support exogenous insulin therapy in achieving the therapeutic goal while reducing the risk of hypoglycaemia and exerting a beneficial effect on body weight. One potential therapeutic option are sodium-glucose co-transporter 2 (SGLT-2) inhibitors. In the present paper, we reviewed the current clinical research on SGLT-2 inhibitors as add-on therapy to insulin in patients with T1DM. This therapy modification contributes to an improvement in metabolic control without increasing the risk of severe hypoglycaemia and with a beneficial effect on body weight, translating to improved compliance, quality of life, and patient satisfaction with treatment. However, due to possible adverse effects including euglycaemic diabetic ketoacidosis, the decision to use SGLT-2 inhibitors in patients with T1DM should be made with caution, and patients require proper education regarding the prevention and treatment of acidosis. (Clin Diabetol 2020; 9; 3: 189-192)

Key words: type 1 diabetes, adjunct therapy, SGLT-2 inhibitors

Address for correspondence: dr n. med. Edyta Cichocka

Szpital Kliniczny nr 1, Katedra i Klinika Chorób Wewnętrznych Diabetologii i Nefrologii w Zabrzu

Śląski Uniwersytet Medyczny w Katowicach

ul. 3 Maja 13/15, 41-800 Zabrze

Phone: +48530032 206

e-mail: ecichocka@sum.edu.pl

Translation: dr n. med. Piotr Jędrusik

Clinical Diabetology 2020, 9, 3, 189-192

DOI: $10.5603 /$ DK.2020.0013

Received: 21.01.2020

Accepted: 09.03.2020

\section{Introduction}

Due to the pathophysiological mechanism of absolute insulin deficiency that mediates the development of type 1 diabetes mellitus (T1DM), the affected patients require insulin substitution therapy along with its all inconveniences. Despite advances in insulin therapy over the last hundred years and introduction of glucose monitoring systems, many patients still do not attain optimal blood glucose and metabolic control and thus are at risk of more rapid development of chronic disease complications. In addition, even with adequate metabolic control, the risk of cardiovascular mortality in patients with T1DM is increased nearly 3-fold [1].

According to the Diabetes Poland guidelines, the recommended treatment model for T1DM is intensive functional insulin therapy using multiple subcutaneous insulin injections or continuous subcutaneous insulin infusion by a personal insulin pump [2]. However, this therapy continues to be associated with a risk of hypoglycaemia, which often makes the optimal blood glucose control more challenging, increases treatment costs and reduces compliance, which ultimately reduces the quality of life. In addition, overweight or obesity and metabolic syndrome coexist in an increasing number of patients with T1DM. In young patients with T1DM, insulin sensitivity is reduced compared to their healthy peers with similar body weight, physical activity level, and body fat content. Exogenous insulin therapy promotes further increase in body weight, which increases insulin requirement, thus creating a pathophysiological vicious circle, and may increase atherogenesis, accelerating the development of late diabetes complications including cardiovascular disease. All these factors result in an increasing interest in adjunct therapies to support exogenous insulin therapy in achieving the therapeutic goal while reducing the risk of hypoglycaemia and exerting a beneficial effect on body weight $[3,4]$. 
Effects of sodium-glucose co-transporter 2 inhibitors in type 1 diabetes mellitus

One potential therapeutic option are sodium-glucose co-transporter 2 (SGLT-2) inhibitors. Inhibition of SGLT-2 leads to a number of beneficial effects including urinary caloric loss (leading to a reduced insulin requirement), body weight reduction, increased insulin sensitivity, blood pressure lowering, and reduced progression of albuminuria and diabetic nephropathy [5], all delaying the development of chronic complications of diabetes. SGLT-2 inhibitors are also effective in reducing the cardiovascular risk in patients with type 2 diabetes mellitus. It was also shown that adding a SGLT-2 inhibitor reduces the risk of hypoglycaemia. Of note, SGLT-2 inhibitors act independently of insulin. Interestingly, SGLT-2 inhibitors are also believed to exert a protective effect on beta cell function, extending their insulin-secreting function [6].

Possible adverse effects of adjunct SGLT-2 inhibitor therapy in T1DM should be taken into account, of which clinically most important are urogenital infections and in particular euglycaemic diabetic ketoacidosis (EDKA). These effects may not only interfere with the therapeutic process but also call for a careful patient selection for such therapy. EDKA is clearly a controversial issue. It is promoted by a reduced carbohydrate availability coupled with a reduced insulin dose. SGLT-2 inhibition increases glucosuria which leads to a reduced plasma insulin level, while the amount of exogenous insulin is reduced and at the same time glucagone level is increased. A lower insulin to glucagone ratio stimulates ketogenesis and lipolysis (with circulating free fatty acid levels increased by $40 \%$ during a meal), which leads to increased lipid oxidation (on average by 20\%) at the expense of carbohydrate oxidation [7]. Factors triggering EDKA include infections, reduced food and fluid intake, reduced insulin dose, and alcohol intake. Pathophysiologically, EDKA is similar to diabetic ketoacidosis (DKA), except for glucosuria induced by SGLT-2 inhibitors which 'artificially' lowers blood glucose level.

\section{Overview of clinical studies using SGLT-2 inhibitors in in type 1 diabetes mellitus}

Currently, more and more reliable data indicate the efficacy of SGLT-2 inhibitors as adjunct therapy in T1DM.

The first SGLT-2 inhibitor approved for T1DM was dapagliflozin, the efficacy and safety of which was assessed in the multicentre, randomized, double-blind, placebo-controlled DEPICT-1 and DEPICT-2 (Efficacy and Safety of Dapagliflozin in Patients with Inadequately Controlled Type 1 Diabetes) studies. The first of these studies was performed in Europe and North America, and the other included patients from North and Latin
America, Europe, and Japan. The DEPICT studies assessed the efficacy and safety of a 24-week dapagliflozin treatment in adult patients (18-75 years of age) with chronic inadequate diabetes control (haemoglobin $A_{1 c}\left[\mathrm{HbA}_{1 \mathrm{c}}\right]$ levels 7.5-10.5\%) who received dapagliflozin $5 \mathrm{mg}(\mathrm{n}=259)$, dapagliflozin $10 \mathrm{mg}(\mathrm{n}=259)$, or placebo $(n=260)$ daily. In the study protocol, the patients were advised to reduce the daily insulin dose by not more than $20 \%$ after taking the first dapagliflozin dose. The DEPICT-1 trial showed a significant reduction of $\mathrm{HbA}_{1 \mathrm{c}}$ level (primary endpoint) by $0.42 \%$ in the $5 \mathrm{mg}$ group and $0.45 \%$ in the $10 \mathrm{mg}$ group $(P<0.0001$ for both doses). A reduction was also noted in the daily insulin dose (by $8.8 \%$ and $13.2 \%$, respectively; $\mathrm{P}<0.0001$ for both doses) and body weight (by $2.96 \%$ and $3.72 \%$, respectively; $\mathrm{P}<0.0001$ for both doses). The proportion of patients with $\mathrm{HbA}_{1 \mathrm{c}}$ level reduction by $\geq 0.5 \%$ without severe hypoglycaemia was significantly higher in both dapagliflozin groups compared to placebo. In addition, in the patient subgroup that used continuous glucose monitoring (CGM), addition of dapagliflozin was shown to result in a significant improvement of the mean daily glucose levels, with an increase of the time in blood glucose level target range by $9.1 \%$ in the $5 \mathrm{mg}$ group and $10.1 \%$ in the $10 \mathrm{mg}$ group $(\mathrm{P}<$ $<0.0001$ for both doses). Severe hypoglycaemia occurred in $8 \%, 6 \%$, and $7 \%$ of patients, respectively, in the $5 \mathrm{mg}, 10 \mathrm{mg}$, and placebo groups. Urogenital infections were noted more frequently in the active treatment groups, while the rates of other adverse effects were similar in all study groups. The incidence of DKA was also similar in all study groups. EDKA was reported in only 2 patients in the $10 \mathrm{mg}$ dapagliflozin group. The positive effects of adjunct dapagliflozin therapy were maintained at the end of extended follow-up period (overall 52 weeks), with a significant reduction in $\mathrm{HbA}_{1 \mathrm{c}}$ level and body weight. During this period, overall 9 cases of EDKA were reported, including one in the placebo group [8]. The DEPICT-2 trial was performed in a study population of a similar size and showed consistent results regarding the efficacy of dapagliflozin in the treatment of T1DM. CGM data showed a similar reduction in blood glucose levels in the active treatment groups, and similar reductions were noted in $\mathrm{HbA}_{1 \mathrm{c}}$ levels, body weight, and daily insulin dose [9]. In March 2019, based on the DEPICT-1 and DEPICT-2 study results, the European Medicines Agency (EMA) approved dapagliflozin at the dose of $5 \mathrm{mg}$ daily as an adjunct therapy in patients with T1DM and body mass index $(B M I) \geq 27 \mathrm{~kg} / \mathrm{m}^{2}$ in whom insulin therapy only is not sufficient for optimal metabolic control [10], but this therapy was not approved by the U.S. Food and Drug Administration (FDA). 
Another somewhat unusual drug of this class is sotagliflozin. It has not been approved for the treatment of type 2 diabetes mellitus and is active against both SGLT-2 and SGLT-1. Sotagliflozin has been approved by EMA was for the treatment of T1DM based on the results of the inTandem studies [11] but again, no approval was granted by FDA. The inTandem study program included 3 multicentre, randomized, doubleblind, placebo-controlled trials. The inTandem-2 and inTandem-1 studies evaluated sotagliflozin $200 \mathrm{mg}$ and $400 \mathrm{mg}$ compared to placebo. The primary endpoint was $\mathrm{HbA}_{1 \mathrm{c}}$ level change at 24 weeks of therapy. The first of these studies was conducted in Europe and the other one in North America. Both included similar numbers of patients (sotagliflozin $400 \mathrm{mg}: \mathrm{n}=263$ in the inTandem-2 study, $\mathrm{n}=262$ in the inTandem-1 study; sotagliflozin $200 \mathrm{mg}: \mathrm{n}=261$ and $\mathrm{n}=263$, respectively; placebo: $n=258$ and $n=268$, respectively). In both studies, the protocol called for the greatest reduction in postprandial insulin dosing (by $30 \%$ ), which was likely related to the additional incretin effect of this drug. Both trials showed significant reductions in $\mathrm{HbA}_{1 \mathrm{c}}$ level ( $\mathrm{P}<0.001$ for both doses), body weight (by $2-3.5 \mathrm{~kg}, \mathrm{P}<0.001$ for both doses), and daily insulin dose (by $6.2-9.7 \%, \mathrm{P}<0.001$ for both doses). In the inTandem-2 study, the proportion of patients with achieved $\mathrm{HbA} 1 \mathrm{c}$ level < $7 \%$ was $27.2 \%, 27.8 \%$, and $15.5 \%$, respectively, in the sotagliflozin $200 \mathrm{mg}$, sotagliflozin $400 \mathrm{mg}$, and placebo groups. In the inTandem-1 study, these proportions were $30 \%, 35.5 \%$, and $20.9 \%$, respectively. In patients using CGM systems, adjunct therapy with sotagliflozin was shown to increase the time in blood glucose level target range by $5.4 \%$ and $11.7 \%$, respectively, for the $200 \mathrm{mg}$ and $400 \mathrm{mg}$ doses $(P=0.026$ for the $200 \mathrm{mg}$ dose; $P<0.001$ for the 400 mg dose). These positive effects on $\mathrm{HbA}_{1 \mathrm{c}}$ levels, body weight, and daily insulin requirement were maintained at 52 weeks of follow-up in both sotagliflozin groups, and the reported satisfaction with treatment increased significantly. The rates of severe hypoglycaemia were lower, while diarrhoea and fungal genital infections were more common in the sotagliflozin groups. The rate of DKA at 52 weeks in the inTandem-2 study was $2.3 \%, 3.4 \%$, and $0 \%$, respectively, in the sotagliflozin $200 \mathrm{mg}$, sotagliflozin $400 \mathrm{mg}$, and placebo groups. In the inTandem 1 study, these rates were 3.4\%, 4.2\%, and $0.4 \%$, respectively. Of 36 cases of DKA reported in these two studies combined, 13 cases occurred with blood glucose levels $<250 \mathrm{mg} / \mathrm{dL}$. The inTandem 3 study showed that addition of sotagliflozin $400 \mathrm{mg}$ contributed to an improved metabolic control, with a significantly higher proportion of patients with $\mathrm{HbA}_{1 \mathrm{c}}$ level $<7.0 \%$ at 24 weeks of follow-up (28.6\% vs. $15.2 \%$,
$P<0.001)$. In addition, the active treatment was associated with positive effects regarding the reduction of $\mathrm{HbA}_{1 \mathrm{c}}$ level $(-0.46 \%)$, body weight $(-2.98 \mathrm{~kg})$, systolic blood pressure $(-3.5 \mathrm{~mm} \mathrm{Hg})$, and daily insulin dose ( -2.8 units daily) ( $\mathrm{P} \leq 0.002$ for all comparisons). The rate of severe hypoglycaemia was similar in both groups (3.0\% vs. $2.4 \%$ in the placebo group). The rate of ketoacidosis was higher in the sotagliflozin group ( $3.0 \%$ vs. $0.6 \%$ in the placebo group), while the rates of other adverse effects were similar in both groups [12].

Long-term safety and efficacy in the treatment of T1DM has also been documented for empagliflozin. The EASE-2 and EASE-3 (Empagliflozin as Adjunctive to inSulin thErapy) studies evaluated the effect of add-on empagliflozin therapy on $\mathrm{HbA}_{1 c}$ levels in adult patients with chronic inadequate T1DM control $\left(\mathrm{HbA}_{1 \mathrm{c}}\right.$ level 7.5-10\%). The EASE-2 trial studied empagliflozin $10 \mathrm{mg}(\mathrm{n}=243)$ and $25 \mathrm{mg}(\mathrm{n}=244)$ vs. placebo ( $n=243$ ), and the EASE-3 trial studied empagliflozin $2.5 \mathrm{mg}(\mathrm{n}=241), 10 \mathrm{mg}(\mathrm{n}=248)$ and $25 \mathrm{mg}(\mathrm{n}=245)$ vs. placebo $(n=241)$.

Study participants were advised to reduce the daily insulin dose by $10 \%$ at the trial initiation. At 26 weeks, a significant reduction of $\mathrm{HbA}_{1 \mathrm{c}}$ level was noted for all empagliflozin doses compared to placebo $(P<0.0001)$. Both trials showed a reduction of body weight and blood glucose level variation, and an increase in the CGM time in range. Systolic blood pressure and daily insulin requirement were also reduced. In addition, the EASE-2 study showed that the positive effects of empagliflozin were maintained during a longer follow-up of 52 weeks. Severe hypoglycaemia occurred in 1.2\%, $4.1 \%, 2.7 \%$, and $3.1 \%$ of patients receiving empagliflozin $2.5 \mathrm{mg}, 10 \mathrm{mg}, 25 \mathrm{mg}$, or placebo, respectively. The rate of genital infections was insignificantly higher in the active treatment groups. DKA was reported in $0.8 \%, 4.3 \%, 3.3 \%$, and $1.2 \%$ of patients in the empagliflozin $2.5 \mathrm{mg}, 10 \mathrm{mg}, 25 \mathrm{mg}$, and placebo groups, respectively. These studies indicated that the $2.5 \mathrm{mg}$ dose was both effective at improving metabolic control in T1DM and safe, as it was not associated with an increased risk of severe hypoglyceamia and EDKA [13]. Of note, this dose is not sufficiently effective in the treatment of type 2 diabetes mellitus.

Similar results of phase 3 clinical trials are currently not available for canagliflozin and ertugliflozin. Smaller phase 2 clinical trials with canagliflozin showed that both $100 \mathrm{mg}$ and $300 \mathrm{mg}$ doses were effective in reducing $\mathrm{HbA}_{1 \mathrm{c}}$ level, body weight, and daily insulin requirement [14].

\section{Summary}

In view of the studies reviewed above, SGLT-2 inhibitors seem an effective and relatively safe thera- 
peutic option as an adjunct therapy in T1DM. Most benefits from such therapy may be expected in patients with chronically uncontrolled diabetes, abnormal body weight (overweight or obesity), and those using relatively large insulin doses. As noted above, the risk of ketoacidosis including EDKA is a limitation that necessitates careful patient selection for adjunct SGLT2 inhibitor therapy in T1DM. Poor candidates for such therapy include patients with risky behaviours including excessive alcohol intake, use of psychoactive substances/ /illicit drugs, and use of a low-calorie low-carbohydrate diet. Inadequate access to a physician is also a contraindication for adding a SGLT-2 inhibitor in patients with T1DM. According to the American Diabetes Association expert consensus published in February 2019, patients with $\mathrm{HbA}_{1 \mathrm{c}}$ levels $>10 \%$ are also not candidates for SGLT-2 inhibitor therapy due to a high rate of ketoacidosis in this group (> 15\% during a 5 -year follow-up) even without SGLT-2 inhibitor therapy [15].

The patients must be educated not to reduce the insulin dose by more than 10-20\% when initiating SGLT-2 inhibitor therapy. The smallest effective dose of any drug of this class should be used. In addition, the drug should be immediately withdrawn in acute conditions associated with a possible DKA trigger such as fasting, infection or other acute illness. The drug should also be withdrawn 72 hours before elective surgery. Patients treated with SGLT-2 inhibitors require monitoring of not only blood glucose levels but also the presence of ketone bodies in serum and urine. Of note, EDKA is not associated with the warning of symptomatic hyperglycaemia, and thus patients should be alert to such symptoms as nausea, vomiting, lack of appetite, fatigue, and dyspnoea, occurring even with blood glucose levels $<250 \mathrm{mg} / \mathrm{dL}$. The management of EDKA associated with SGLT-2 inhibitor use is summarized by the STICH mnemonic (STopping SGLT-2 inhibitor therapy, Injecting insulin, consuming Carbohydrates, Hydrating, monitoring ketones) [16].

In summary, adding a SGLT-2 inhibitor as an adjunct to insulin therapy in T1DM has been shown to be an effective therapeutic approach. Such therapy modification contributes to a better disease control as evidenced by lower $\mathrm{HbA}_{1 \mathrm{c}}$ levels and body weight and improved CGM parameters without an increased risk of severe hypoglycaemia. It also seems that these benefits translate to improved compliance, quality of life, and patient satisfaction with treatment. However, not all patients with T1DM are candidates for such therapy due to possible adverse effects including the most dangerous complication of (euglycaemic) DKA. Patients in whom SGLT-2 inhibitor therapy is considered must be well educated, with an emphasis on the identification of DKA symptoms and triggers, and the management of this condition should it occur.

\section{Conflict of interests}

The authors declare no conflicts of interests.

\section{REFERENCES}

1. Lind M, Svensson AM, Rosengren A, et al. Glycemic control and excess mortality in type 1 diabetes. N Engl J Med. 2014; 371(21): 1972-1982, doi: 10.1056/NEJMoa1408214, indexed in Pubmed: 25409370.

2. Araszkiewicz A, Bandurska-Stankiewicz E, Budzyński A, et al. 2019 Guidelines on the management of diabetic patients. A position of Diabetes Poland. Clinical Diabetology. 2019; 8(1): 1-95, doi: 10.5603/dk.2019.0001.

3. Bjornstad P, Snell-Bergeon JK, Nadeau KJ, et al. Insulin sensitivity and complications in type 1 diabetes: New insights. World J Diabetes. 2015; 6(1): 8-16, doi: 10.4239/wjd.v6.i1.8, indexed in Pubmed: 25685274.

4. Margeirsdottir HD, Stensaeth $\mathrm{KH}$, Larsen JR, et al. Early signs of atherosclerosis in diabetic children on intensive insulin treatment: a population-based study. Diabetes Care. 2010; 33(9): 2043-2048, doi: 10.2337/dc10-0505, indexed in Pubmed: 20530748.

5. Poudel RR. Renal glucose handling in diabetes and sodium glucose cotransporter 2 inhibition. Indian J Endocrinol Metab. 2013; 17(4): 588-593, doi: 10.4103/2230-8210.113725, indexed in Pubmed: 23961473.

6. Asahara Si, Ogawa W. SGLT2 inhibitors and protection against pancreatic beta cell failure. Diabetol Int. 2018; 10(1): 1-2, doi: 10.1007/s13340-018-0374-y.

7. Rosenstock J, Ferrannini E. Euglycemic Diabetic Ketoacidosis: A Predictable, Detectable, and Preventable Safety Concern With SGLT2 Inhibitors. Diabetes Care. 2015; 38(9): 1638-1642, doi: 10.2337/dc15-1380, indexed in Pubmed: 26294774.

8. Dandona P, Mathieu C, Phillip M, et al. DEPICT-1 Investigators. Efficacy and Safety of Dapagliflozin in Patients With Inadequately Controlled Type 1 Diabetes: The DEPICT-1 52-Week Study. Diabetes Care. 2018; 41(12): 2552-2559, doi: 10.2337/dc18-1087, indexed in Pubmed: 30352894.

9. Mathieu C, Dandona P, Gillard P, et al. DEPICT-2 Investigators. Efficacy and Safety of Dapagliflozin in Patients With Inadequately Controlled Type 1 Diabetes (the DEPICT-2 Study): 24-Week Results From a Randomized Controlled Trial. Diabetes Care. 2018; 41(9): 1938-1946, doi: 10.2337/dc18-0623, indexed in Pubmed: 30026335

10. https://www.ema.europa.eu/en/documents/press-release/firstoral-add-treatment-insulin-treatment-certain-patients-type1-diabetes en.pdf.

11. https://www.ema.europa.eu/en/documents/smop-initial/chmpsummary-positive-opinion-zynquista_en.pdf.

12. Danne T, Cariou B, Buse JB, et al. Improved Time in Range and Glycemic Variability With Sotagliflozin in Combination With Insulin in Adults With Type 1 Diabetes: A Pooled Analysis of 24-Week Continuous Glucose Monitoring Data From the inTandem Program. Diabetes Care. 2019; 42(5): 919-930, doi: 10.2337/ dc18-2149, indexed in Pubmed: 30833371.

13. Rosenstock J, Marquard J, Laffel LM, et al. Empagliflozin as Adjunctive to Insulin Therapy in Type 1 Diabetes: The EASE Trials. Diabetes Care. 2018; 41(12): 2560-2569, doi: 10.2337/dc181749, indexed in Pubmed: 30287422.

14. Henry R, Thakkar P, Tong C, et al. Efficacy and Safety of Canagliflozin, a Sodium-Glucose Cotransporter 2 Inhibitor, as Add-on to Insulin in Patients With Type 1 Diabetes. Diabetes Care. 2015; 38(12): 2258-2265, doi: 10.2337/dc15-1730.

15. Danne T, Garg S, Peters AL, et al. International Consensus on Risk Management of Diabetic Ketoacidosis in Patients With Type 1 Diabetes Treated With Sodium-Glucose Cotransporter (SGLT) Inhibitors. Diabetes Care. 2019; 42(6): 1147-1154, doi: 10.2337/ dc18-2316, indexed in Pubmed: 30728224

16. Patel KS, Carbone AM, Patel K, et al. Sodium-Glucose Cotransporters as Potential Therapeutic Targets in Patients With Type 1 Diabetes Mellitus: An Update on Phase 3 Clinical Trial Data. Ann Pharmacother. 2019; 53(12): 1227-1237, doi: 10.1177/1060028019859323, indexed in Pubmed: 31226886 